\title{
Potential mechanisms of spontaneous regression in patients with B-cell lymphoma; the significance of co-stimulatory molecules in lymphoma cells
}

Keywords: spontaneous regression, remission, lymphoma

\section{TO THE EDITOR}

Spontaneous regression (SR) of malignant tumors has been observed in several types of tumors including lymphoma, kidney cancer, melanoma, and neuroblastoma. ${ }^{1}$ SR is currently of interest for many clinicians because of an increased number of methotrexate-related lymphoproliferative disorders, and because SR is observed in many patients after withdrawal of methotrexate. ${ }^{2}$ In terms of a mechanism, anti-tumor immune responses by host $\mathrm{T}$ lymphocytes reacting against tumor cells are believed to be involved in SR, ${ }^{3}$ and several cases have recently been reported. In a recent issue of journal of clinical and experimental hematopathology $(J C E H)$, Tanaka et al. described a case of diffuse large B-cell lymphoma (DLBCL) with SR. ${ }^{4}$ A 35-year-old man had multiple mesenteric lymphadenopathy and a thickened small intestine wall, and was diagnosed with DLBCL (germinal center origin) without infection with Epstein-Barr virus (EBV) following laparoscopic lymph node biopsy. However, symptoms were improved and abnormal accumulation of fluorodeoxyglucose was disappeared 3 months after the biopsy. In addition, Abe et al. previously reported in $J C E H$ a case of DLBCL harboring EBV infection with SR and reviewed some published SR cases of aggressive nonHodgkin's lymphoma. ${ }^{5}$ SR has also been seen in low-grade lymphoma. Matsuo et al. described a case of bilateral conjunctival extranodal marginal zone B-cell lymphoma of mucosa-associated lymphoid tissue with SR. ${ }^{6}$ Ye et al. just recently published four cases of SR in patients with mantle cell lymphoma. ${ }^{7}$ SR is preferentially seen in extranodal lymphoma including in the intestinal tract.

SR (also referred as "healing") is also observed in patients with non-hematological malignant tumors such as lung cancer, kidney cancer, breast cancer, and melanoma. ${ }^{8-11}$ These solid cancers are known as immunogenic tumors because of increased expression of neoantigens, and antitumor therapy using immune checkpoint blockade antibodies and cytokines such as interferons have been used for these cancers. $^{12,13}$ However, mutation burdens of high-grade lymphomas are less than those of melanoma and lung cancers, indicating that unknown mechanisms are involved in SR in lymphoma cases. ${ }^{14}$

CD80 and CD86 are well-known co-stimulatory molecules expressed on antigen-presenting cells including B cells.
CD80 is expressed on lymphoma cells in $90 \%$ of DLBCL cases, ${ }^{15}$ and the expression of both CD80 and CD86 is widely seen in leukemia or lymphoma cell lines in the NCI-60 cancer panel database [GEO data set, GDS4296]. As shown in figure 1, CD80 expression was observed in B-cell lymphoma and B-cell lymphoma cell lines. In addition, human leukocyte antigen (HLA)-DR, one of major histocompatibility complex (MHC) class II molecules, is also expressed in $65 \%$ of DLBCL cases, and HLA-DR-positive cases show a significantly better clinical course. ${ }^{16}$ Given that lymphoma cells in DLBCL expressing co-stimulatory molecules such as CD80/ CD86 and MHC class II molecules, lymphoma cells may have the higher immunogenic potential than other solid tumors. In support of this, Allison (received the Nobel Prize in 2018) et al. previously found that ectopic expression of $\mathrm{CD} 80$ on tumor cells induces $\mathrm{T}$ cell-mediated rejection in murine models by not CD4-positive $\mathrm{T}$ cells but CD8-positive T cells. ${ }^{17}$ In addition, clinical trials with tumor cell vaccines using CD80-transfected autologous or allogenic tumor cells were performed for kidney cancer, lung cancer, and acute myeloid leukemia. ${ }^{18}$ As a result, some patients who enrolled in these trials showed significant tumor reduction. ${ }^{19-21}$ Although the overall response rate was limited, these findings indicate that CD80-expressing tumor cells could enhance anti-tumor immune responses. The interaction between CD80/CD86 and CD28 activates tumor-specific T cells to produce interleukin (IL)-2, which in turn triggers T cell proliferation in autocrine and paracrine manners in tumor microenvironment (Figure 2). Given that the interaction between CD80/86 and CTLA-4 results in T cell inactivation, therapies to block CTLA-4-mediated immunosuppression may improve this immune response.

Regarding EBV-infected lymphoma or lymphoproliferation, anti-EBV immune responses are believed to induce antilymphoma immune responses and SR. ${ }^{22}$ However, EBVtransformed B lymphocytes and EBV-infected lymphoma cells produce IL-12, which is a cytokine to promote cellular immunity and is produced after CD40 ligation. ${ }^{23}$ IL-12 production from lymphoma cells may be involved in SR in EBV-infected lymphoma or lymphoproliferative disorders.

Traumatic stress or injury including biopsy is considered to be a trigger for SR, and occasionally, administration of corticosteroids, anti-lymphoma drugs, or infection may cause the initiation of SR. ${ }^{1-3}$ We propose a possibility that, after 
A

B
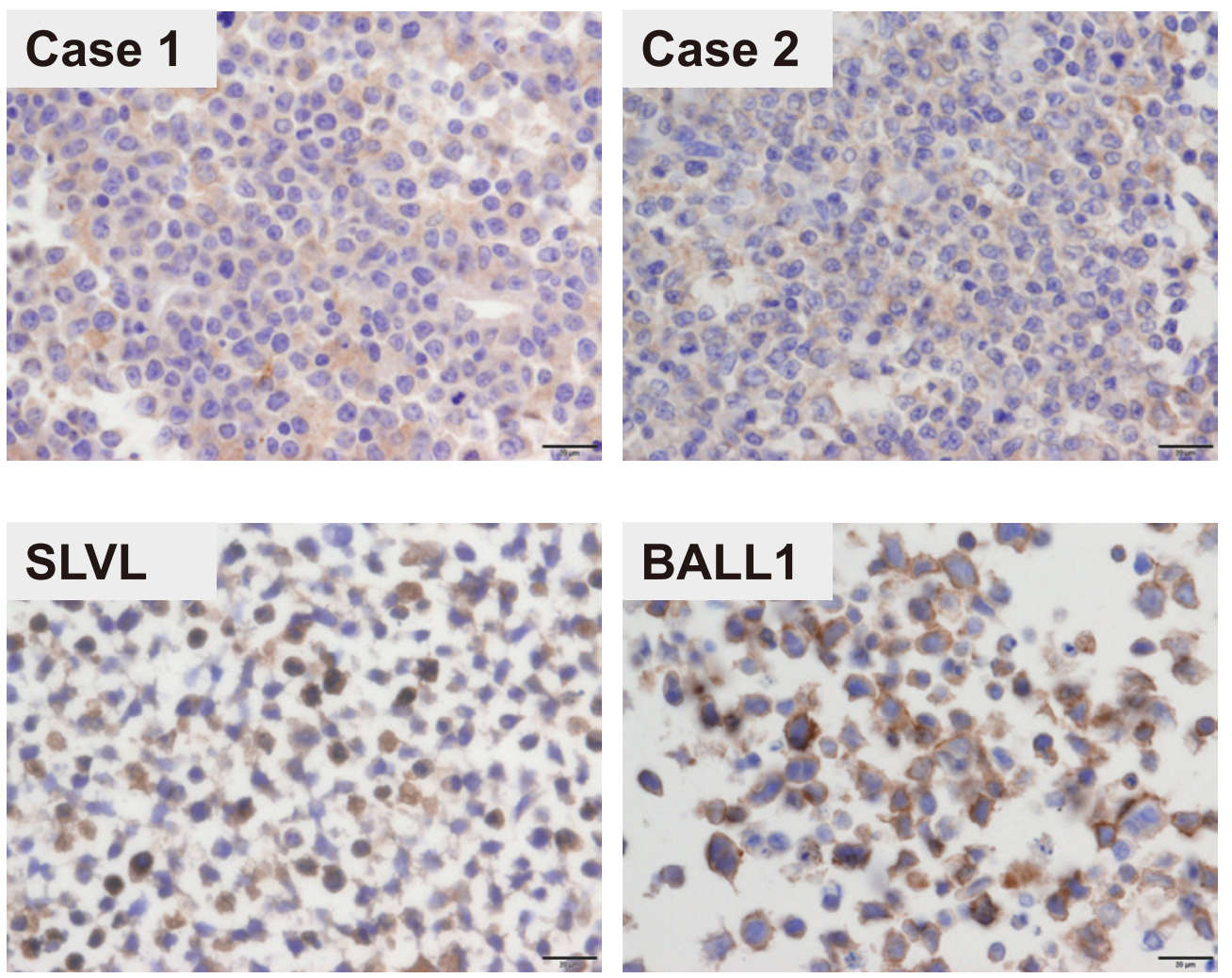

Fig. 1. CD80 expression in lymphoma tissues and cell lines. $(\boldsymbol{A})$ The immunostaining using anti-CD80 monoclonal antibody (clone EPR1157, Abcam) was performed as described previous methods. ${ }^{30}$ Lymphoma cells were weakly positive for CD80 in diffuse large B-cell lymphoma $(\boldsymbol{A})$, and strongly positive in two B-cell lymphoma cell lines (SLVL and BALL1) (B). Scale bar; $20 \mu \mathrm{m}$.

A
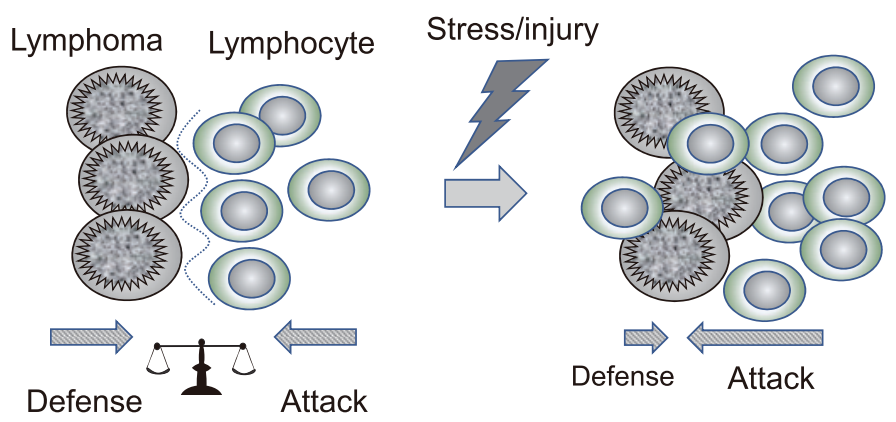

B

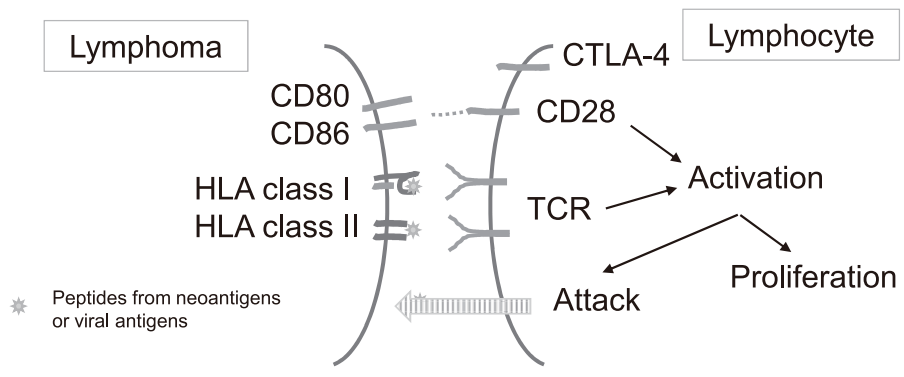

Fig. 2. Scheme of the suggested mechanisms of spontaneous regression (SR). (A) In the growing phase of lymphoma, lymphoma cells are protected from microenvironment that includes cytotoxic T lymphocytes. Stress or injury disrupts the microenvironment, and immune reactions between $\mathrm{T}$ lymphocytes and lymphoma cells can be initiated. (B) Co-stimulatory molecules such as CD80/CD86 stimulate lymphoma-specific T cell response. Activated T lymphocytes proliferate and attack lymphoma cells, which present neoantigens or viral antigens with HLA class I or class II molecules. 
lymphoma cells are exposed to anti-lymphoma T lymphocytes by physical disruption of the microenvironment, immune reaction between lymphoma cells and lymphomaspecific T lymphocytes may be initiated. Damage-associated molecular patterns are also considered to be involved in this immune reaction by activating the STING pathway in antigen-presenting cells. ${ }^{24}$

Recent advances of immunotherapy indicated the significance of programmed death-1 (PD-1) and its ligands such as PD-L1 and PD-L2. PD-L1-expression in lymphoma cells was seen in $11 \%$ of cases and reportedly associated to poor clinical course in DLBCL. ${ }^{25}$ PD-L1 expression in lymphoma cells were potentially mediated by Stat 3 activation which were suggested to be induced by macrophage-derived factors. ${ }^{26,27}$ Indoleamine 2,3-dioxygenase (IDO) which has immunosuppressive functions due to enzymatic activities catalyzing the essential amino acid L-tryptophan was also expressed on $32 \%$ of B-cell lymphoma cases and IDO expression was associated to poor outcome. ${ }^{28}$ These immunosuppressive molecules are also expressed on myeloid cells such as tumor associated macrophages. ${ }^{29}$ Down-regulation of these factors might be linked to SR in lymphoma cases.

In conclusion, the expression of CD80/CD86 on lymphoma cells is potentially associated with activation of antilymphoma $\mathrm{T}$ cell responses and clinical SR. HLA-DR expression on lymphoma cells may also influence activation of lymphoma-specific CD4-positive helper T cells in the microenvironment. As a therapeutic strategy, anti-CTLA-4 antibody rather than anti-PD-1/PD-L1 antibody may be helpful to enhance anti-lymphoma $\mathrm{T}$ cell response in cases of CD80/CD86-positive lymphoma.

\section{CONFLICT OF INTEREST}

All authors have no financial competing interests to declare.

\section{REFERENCES}

1 Ghatalia P, Morgan CJ, Sonpavde G. Meta-analysis of regression of advanced solid tumors in patients receiving placebo or no anti-cancer therapy in prospective trials. Crit Rev Oncol Hematol. 2016; 98 : 122-136.

2 Tokuhira M, Tamaru J, Kizaki M. Clinical management for other iatrogenic immunodeficiency-associated lymphoproliferative disorders. J Clin Exp Hematop. 2019; 59 : 72-92.

3 Saito S, Suzuki K, Yoshimoto K, et al. Restoration of Decreased T Helper 1 and CD8+ T Cell Subsets Is Associated With Regression of Lymphoproliferative Disorders Developed During Methotrexate Treatment. Front Immunol. 2018; 9 : 621.

4 Tanaka Y, Ishihara M, Miyoshi H, et al. Spontaneous regression of diffuse large B-cell lymphoma in the small intestine with multiple lymphadenopathy. J Clin Exp Hematop. 2019; 59 : 17-21.

5 Abe R, Ogawa K, Maruyama Y, Nakamura N, Abe M. Spontaneous regression of diffuse large B-cell lymphoma harbouring Epstein-Barr virus: a case report and review of the liter- ature. J Clin Exp Hematop. 2007; 47 : 23-26.

6 Matsuo T, Ichimura K, Yoshino T. Spontaneous regression of bilateral conjunctival extranodal marginal zone B-cell lymphoma of mucosa-associated lymphoid tissue. J Clin Exp Hematop. 2007; 47 : 79-81.

7 Ye H, Desai A, Gong T, et al. Spontaneous regression of mantle cell lymphoma: a report of four cases. Cancer Commun (Lond). 2018; $38: 30$.

8 Kappauf H, Gallmeier WM, Wünsch PH, et al. Complete spontaneous remission in a patient with metastatic non-small-cell lung cancer. Case report, review of the literature, and discussion of possible biological pathways involved. Ann Oncol. 1997; 8 : 1031-1039.

9 Janiszewska AD, Poletajew S, Wasiutyński A. Spontaneous regression of renal cell carcinoma. Contemp Oncol (Pozn). 2013; $17:$ 123-127.

10 Bramhall RJ, Mahady K, Peach AHS. Spontaneous regression of metastatic melanoma - Clinical evidence of the abscopal effect. Eur J Surg Oncol. 2014; 40 : 34-41.

11 Horii R, Akiyama F, Kasumi F, Koike M, Sakamoto G. Spontaneous "healing" of breast cancer. Breast Cancer. 2005; $12: 140-144$.

12 Vasquez M, Tenesaca S, Berraondo P. New trends in antitumor vaccines in melanoma. Ann Transl Med. 2017; $5: 384$.

13 Turajlic S, Litchfield $\mathrm{K}, \mathrm{Xu} \mathrm{H}$, et al. Insertion-and-deletionderived tumour-specific neoantigens and the immunogenic phenotype: a pan-cancer analysis. Lancet Oncol. 2017; 18 : 1009-1021.

14 Lawrence MS, Stojanov P, Polak P, et al. Mutational heterogeneity in cancer and the search for new cancer-associated genes. Nature. 2013; 499 : 214-218.

15 Dakappagari N, Ho SN, Gascoyne RD, et al. CD80 (B7.1) is expressed on both malignant $\mathrm{B}$ cells and nonmalignant stromal cells in non-Hodgkin lymphoma. Cytometry B Clin Cytom. 2012; $82: 112-119$.

16 Rimsza LM, Roberts RA, Miller TP, et al. Loss of MHC class II gene and protein expression in diffuse large B-cell lymphoma is related to decreased tumor immunosurveillance and poor patient survival regardless of other prognostic factors: a follow-up study from the Leukemia and Lymphoma Molecular Profiling Project. Blood. 2004; 103 : 4251-4258.

17 Townsend SE, Su FW, Atherton JM, Allison JP. Specificity and longevity of antitumor immune responses induced by B7-transfected tumors. Cancer Res. 1994; 54 : 6477-6483.

18 Zang X, Allison JP. The B7 family and cancer therapy: costimulation and coinhibition. Clin Cancer Res. 2007; 13 : 5271-5279.

19 Antonia SJ, Seigne J, Diaz J, et al. Phase I trial of a B7-1 (CD80) gene modified autologous tumor cell vaccine in combination with systemic interleukin-2 in patients with metastatic renal cell carcinoma. J Urol. 2002; 167 : 1995-2000.

20 Chan L, Hardwick NR, Guinn B, et al. An immune edited tumour versus a tumour edited immune system: prospects for immune therapy of acute myeloid leukaemia. Cancer Immunol Immunother. 2006; 55 : 1017-1024.

21 Raez LE, Cassileth PA, Schlesselman JJ, et al. Allogeneic vaccination with a B7.1 HLA-A gene-modified adenocarcinoma cell line in patients with advanced non-small-cell lung cancer. J 
Clin Oncol. 2004; 22 : 2800-2807.

22 Ikeda T, Gion Y, Yoshino T, Sato Y. A review of EBV-positive mucocutaneous ulcers focusing on clinical and pathological aspects. J Clin Exp Hematop. 2019; 59 : 64-71.

23 Airoldi I, Guglielmino R, Carra G, et al. The interleukin-12 and interleukin-12 receptor system in normal and transformed human B lymphocytes. Haematologica. 2002; 87 : 434-442.

24 Temizoz B, Kuroda E, Ishii KJ. Vaccine adjuvants as potential cancer immunotherapeutics. Int Immunol. 2016; 28 : 329-338.

25 Kiyasu J, Miyoshi H, Hirata A, et al. Expression of programmed cell death ligand 1 is associated with poor overall survival in patients with diffuse large B-cell lymphoma. Blood. 2015; $126: 2193-2201$.

26 Li L, Zhang J, Chen J, et al. B-cell receptor-mediated NFATc1 activation induces IL-10/STAT3/PD-L1 signaling in diffuse large B-cell lymphoma. Blood. 2018; 132 : 1805-1817.

27 Ma C, Horlad H, Pan C, et al. Stat3 inhibitor abrogates the expression of PD-1 ligands on lymphoma cell lines. J Clin Exp Hematop. 2017; $57: 21-25$.

28 Ninomiya S, Hara T, Tsurumi H, et al. Indoleamine 2,3-dioxygenase in tumor tissue indicates prognosis in patients with diffuse large B-cell lymphoma treated with R-CHOP. Ann Hematol. 2011; 90 : 409-416.

29 Miyasato Y, Takashima Y, Takeya H, et al. The expression of PD-1 ligands and IDO1 by macrophage/microglia in primary central nervous system lymphoma. J Clin Exp Hematop. 2018; $58: 95-101$.
30 Nakagawa T, Ohnishi K, Kosaki Y, et al. Optimum immunohistochemical procedures for analysis of macrophages in human and mouse formalin fixed paraffin-embedded tissue samples. J Clin Exp Hematop. 2017; 57 : 31-36.

Yoshihiro Komohara, ${ }^{1,3)}$ Mamoru Harada ${ }^{2)}$

${ }^{1)}$ Department of Cell Pathology, Graduate School of Medical Sciences, Kumamoto University, Kumamoto, Japan,

${ }^{2)}$ Department of Immunology, Faculty of Medicine, Shimane

University, Shimane, Japan, ${ }^{3)}$ Center for Metabolic Regulation of Healthy Aging, Kumamoto University, Kumamoto, Japan

Corresponding author: Yoshihiro Komohara, Department of Cell Pathology, Graduate School of Medical Sciences, Kumamoto University, Honjo 1-1-1, Kumamoto, 860-8556, Japan. E-mail: ycomo@kumamoto-u.ac.jp

Received: July 24, 2019.

Revised: September 25, 2019.

Accepted: October 8, 2019.

J-STAGE Advance Published: November 8, 2019

DOI:10.3960/jslrt.19026

Copyright (C) 2019 The Japanese Society for Lymphoreticular Tissue Research (cc) BY-NC-SA This work is licensed under a Creative Commons AttributionNonCommercial-ShareAlike 4.0 International License. 